\title{
Theory of atomic scale quantum dots in silicon: dangling bond quantum dots on silicon surface
}

\author{
Alain Delgado ${ }^{1}$, Marek Korkusinski ${ }^{1,2}$, Pawel Hawrylak ${ }^{1}$ \\ ${ }^{1}$ Department of Physics, University of Ottawa, Ottawa, Canada K1N $6 N 5$ and \\ ${ }^{2}$ National Research Council of Canada, Ottawa, Canada K1A OR6
}

\begin{abstract}
We present a theory and a computational tool, Silicon-QNANO, describing atomic scale quantum dots in silicon. The developed methodology is applied to model dangling bond quantum dots (DBQDs) created on a passivated H:Si- $(100)-(2 \times 1)$ surface by removing a hydrogen atom. The electronic properties of the DBQD are computed by embedding it in a computational box of silicon atoms. The surfaces of the computational box were constructed by using density functional theory as implemented in the ABINIT package. The top layer was reconstructed by the formation of $\mathrm{Si}$ dimers passivated with $\mathrm{H}$ atoms while the bottom layer remained unreconstructed and fully saturated with $\mathrm{H}$ atoms. The computational box Hamiltonian was approximated by a tight-binding (TB) Hamiltonian by expanding the electron wave functions as linear combinations of atomic orbitals and fitting the bandstructure to ab-initio results. The parametrized TB Hamiltonian was used to model large finite $\mathrm{Si}(100)$ boxes (slabs) with number of atoms exceeding present capabilities of ab-initio calculations. The removal of one hydrogen atom from the reconstructed surface resulted in a DBQD state with a wave function strongly localized around the Si atom and the energy in the silicon bandgap. The DBQD could be charged with zero, one, and two electrons. The Coulomb matrix elements were calculated and the charging energy of a two electron complex in a DBQD obtained.
\end{abstract}




\section{INTRODUCTION}

There is currently interest in extending silicon-based microelectronics to quantum technologies, including silicon nanocrystals [1], gated quantum dots [2, 3], and dopants [4, 5]. Recently, several groups have demonstrated the possibility of using scanning tunneling microscopy (STM) to remove hydrogen atoms from a hydrogen passivated $\mathrm{Si}(100)-(2 \times 1)$ surface [6-13]. The removal of a hydrogen atom from the surface creates a dangling bond (DB) in a silicon atom with corresponding energy in the gap of bulk $\mathrm{Si}$, well below the bottom of the conduction band [7, 14]. This DB can be charged in a controlled way with electrons drawn from n-type doped Si substrate [6, 8]. DBs were used for atom-by-atom construction of linear chains and cyclic artificial molecules in silicon [8, 10 13].

When the dangling bond quantum dot (DBQD) is charged and/or manipulated, the quantum structure involves a large number of atoms. The same is true for quantum circuits created with dopants, nanocrystals, and gated silicon quantum dots - the number of silicon atoms involved even in a very small circuit can easily exceed a million. Hence to develop an understanding of atomic scale quantum computing devices in silicon one needs a computational tool suitable for designing circuits made of millions of atoms. Here we describe Silicon-QNANO (Si-QNANO), a QNANO computational platform [15 17] for the design of quantum nanostructures in silicon and apply it to atomic scale DB-based quantum dots on a surface of silicon.

\section{COMPUTATIONAL SILICON BOX WITH RECONSTRUCTED SURFACE}

In this section we describe a finite computational box made of silicon atoms with a reconstructed and passivated top surface on which impurities, defects, dangling bonds, or external gates are implemented. The bottom surface, on the other hand, is constructed to simulate bulk silicon. We start with a small box with a number of silicon atoms suitable for ab-initio calculations. Fig. 1(a) shows the computational supercell used to model such a box, or a slab, here with 8 Si layers. The top Si surface, passivated with H atoms, aims at simulating a real Si surface, followed by a vacuum region in the $[0,0,1]$ direction. This supercell is repeated periodically in the lateral and vertical directions using the lattice vectors $\vec{a}_{1}=a_{1} \hat{\mathbf{x}}, \vec{a}_{2}=a_{2} \hat{\mathbf{y}}$ and $\vec{a}_{3}=a_{3} \hat{\mathbf{z}}$. Here $a_{1}=\sqrt{2} a_{\mathrm{Si}}, a_{2}=\sqrt{2} a_{\mathrm{Si}} / 2$ and $a_{3}=2 a_{\mathrm{Si}}+h_{\mathrm{vac}}$ 


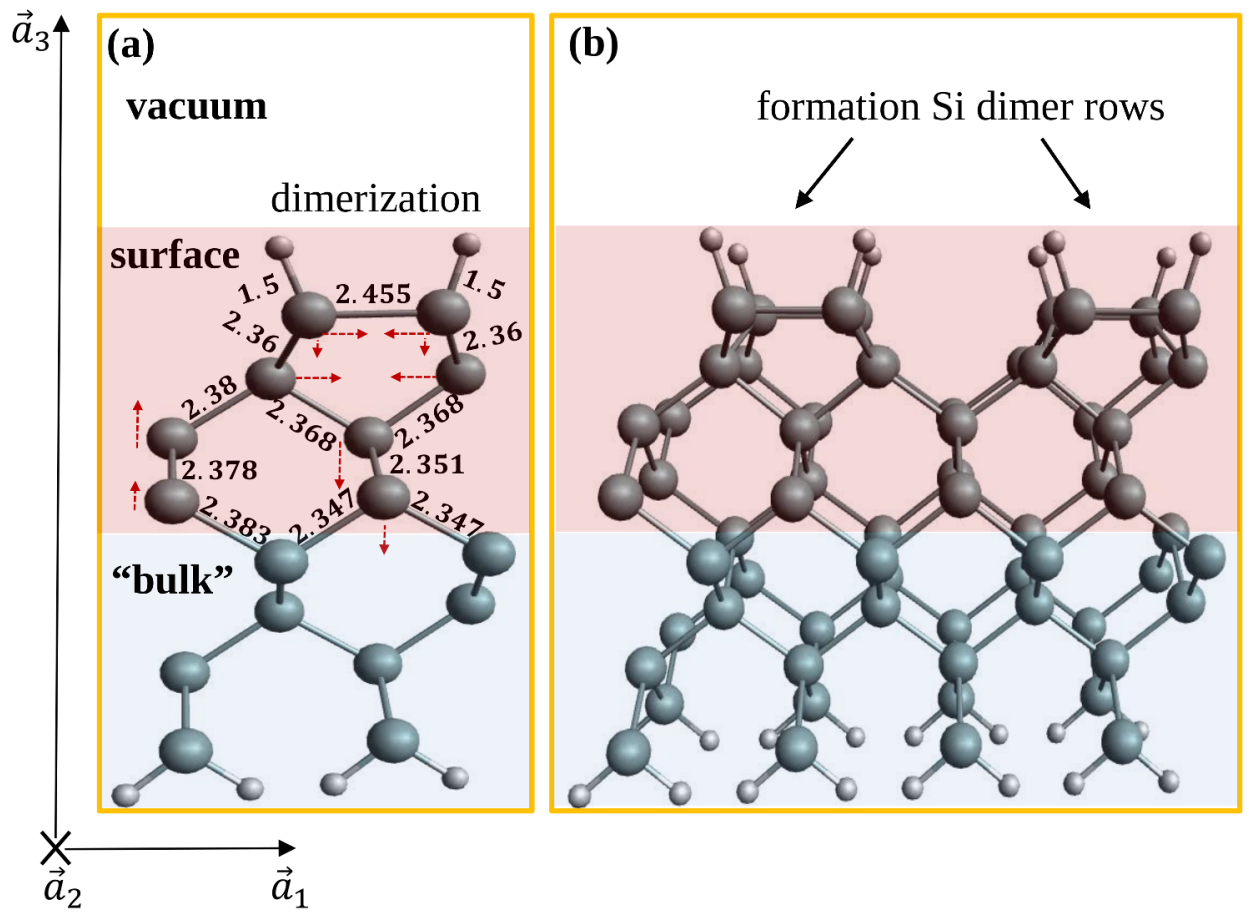

FIG. 1. (color online) (a) Optimized atomic structure of the supercell used to model the top surface reconstruction of a Si-(100) slab. Bond lengths are reported explicitly in Angstroms. Dashed (red) arrows indicate the displacements of $\mathrm{Si}$ atoms respective to their positions in bulk material due to surface reconstruction. (b) A larger slab supercell illustrating the formation of rows of Si dimers on the reconstructed surface of the slab.

where $a_{\mathrm{Si}}$ is the lattice constant of bulk Si and $h_{\mathrm{vac}}$ is the height of the vacuum region.

In order to understand the structure and electronic properties of our Si computational box we perform density functional theory (DFT) calculations using the PBE generalized gradient approximation to the exchange-correlation energy functional [18, 19] as implemented in the ABInIT code [20]. All calculations were performed using a plane wave basis set, truncated with a kinetic energy cutoff of $20 \mathrm{Ha}(544 \mathrm{eV})$. A grid with $6 \times 12 \times 1 \mathrm{k}$-points was used for Brillouin zone integrations using Monkhorst-Pack method [21]. First, we optimized the lattice constant of bulk silicon and obtained $a_{\mathrm{Si}}=5.46 \AA$. Note that this value agrees up to $0.03 \AA$ with the experimentally observed lattice constant of Silicon, $a_{\mathrm{Si}}^{\exp }=5.43 \AA[22]$.

Next we proceed to the surface reconstruction. First, for a given number of Si layers the thickness $h_{\text {vac }}$ of vacuum above the surface was varied until total energy did not depend on it. We found that a vacuum region of $16 \AA$ was enough to suppress interactions between 
periodic images in the $\hat{\mathbf{z}}$ direction. Next, we varied the number of Si layers of the slab and found that 8 layers were sufficient to achieve convergence of the surface energy per surface unit cell. With $8 \mathrm{Si}$ layers there are $16 \mathrm{Si}$ atoms and $6 \mathrm{H}$ atoms in a supercell, as shown in Fig. 1(a).

Surface reconstruction was achieved by minimizing the total energy with respect to the atomic positions of $\mathrm{H}$ and $\mathrm{Si}$ atoms in the top four layers of the slab. Atomic coordinates were adjusted until the maximum interatomic force was less than $0.001 \mathrm{eV} / \AA$. In Fig. 1(a) we illustrate with arrows the displacements of Si atoms with respect to their positions in the bulk material. The positions of Si atoms in the bottom four layers were not optimized as relaxation decreases very rapidly as we move away from the top surface. The bottom surface is unreconstructed and fully passivated with $\mathrm{H}$ atoms to simulate a seamless transition to the bulk material.

The essence of the reconstruction can be understood by comparing the positions and coordination of the two topmost surface Si atoms in the unit cell in Fig. 1(a) to the two Si atoms at the bottom of the cell, simulating bulk material. Each of the two bottom Si atoms is bonded to two other atoms above it, of which only one is seen in Fig. 1)(a). The bonding becomes more apparent when we begin building up the slab, as presented in Fig. 1(b). Moreover, each Si atom of the bottom layer is connected to two $\mathrm{H}$ atoms underneath it. In the full slab geometry, the bottom layer Si atoms are distributed regularly and form the (100) crystal plane as they do in the bulk. In contrast, the surface (top) Si atoms are coordinated differently. Each atom has three Si nearest neighbors, and a single DB, which is saturated by the $\mathrm{H}$ atom. As indicated by the red arrows in Fig. 1 (a), this reconstruction is realized by (i) a shift of atomic positions of the first (top) and, to a lesser extent, the second layer, and (ii) the formation of a new Si-Si bond between the top two Si atoms. We stress that the new bond is remarkably only about $5 \%$ longer than the bulk Si-Si bond. The key qualitative change introduced by the surface reconstruction is that this bond connects only alternate Si pairs, resulting in dimers evident in Fig. 1(b).

\section{TIGHT-BINDING ELECTRONIC-STRUCTURE CALCULATIONS}

In this section we describe the model tight binding (TB) Hamiltonian we use to perform approximate calculations of the Kohn-Sham (KS) quasiparticles of the Si slab described in 
the previous section. The KS quasiparticle Hamiltonian reads:

$$
\hat{H}_{Q P}=\frac{\vec{p}^{2}}{2 m}+V_{\text {atoms }}(\vec{r})+V_{\text {Hartree }}(\vec{r})+V_{\text {xc }}(\vec{r}),
$$

where $V_{\text {atoms }}(\vec{r})$ is the sum of atomic potentials, $V_{\text {Hartree }}(\vec{r})$ is the Hartree potential produced by all electrons, and $V_{\mathrm{xc}}(\vec{r})$ is the exchange-correlation potential. If we carry out the fully self-consistent DFT calculations as in the previous section, the KS Hamiltonian is expressed in terms of atomic, Hartree, and exchange-correlation potentials, themselves functionals of the ground state electronic density. Since we do not know the Hamiltonian for the number of atoms exceeding $a b$ initio capabilities, we parametrize it in a TB form by expanding the electron wave function as a linear combination of atomic orbitals (LCAO) of the type $\alpha$ on the atom at position $\vec{R}$ :

$$
|\phi\rangle=\sum_{\vec{R}, \alpha} c_{\vec{R} \alpha}|\vec{R} \alpha\rangle
$$

In our tight-binding approach we retain ten valence orbitals for each Si atom: one $s$, three $p$, five $d$, and one additional $s^{*}$ orbital that accounts for higher lying states, and by one $s$ orbital on each $\mathrm{H}$ atom. In this basis, the TB Hamiltonian can be written in second quantization as follows:

$$
\hat{H}_{\mathrm{TB}}=\sum_{i=1}^{N_{\mathrm{Si}}+N_{\mathrm{H}}} \sum_{\alpha=1}^{N_{\mathrm{orb}}^{(i)}} \varepsilon_{i \alpha} c_{i \alpha}^{+} c_{i \alpha}+\sum_{i=1}^{N_{\mathrm{Si}}+N_{\mathrm{H}}} \sum_{j=1}^{\mathrm{NN}^{(i)}} \sum_{\alpha=1}^{N_{\mathrm{orb}}^{(i)}} \sum_{\beta=1}^{N_{\mathrm{orb}}^{(j)}} t_{i \alpha, j \beta} c_{i \alpha}^{+} c_{j \beta} .
$$

In Eq. (3), $c_{i \alpha}^{+}\left(c_{i \alpha}\right)$ is the creation (annihilation) operator of an electron on the orbital $\alpha$ localized on the site $i, \varepsilon_{i \alpha}$ is the corresponding on-site energy, and $t_{i \alpha, j \beta}$ describe the hopping of the particle between orbitals on neighboring sites. $N_{\mathrm{Si}}$ and $N_{\mathrm{H}}$ are, respectively, the total number of $\mathrm{Si}$ and $\mathrm{H}$ atoms in the slab, $N_{\mathrm{orb}}^{(i)}$ is the number of atomic orbitals centered on site $i$ and $\mathrm{NN}^{(i)}$ is the number of nearest neighbors of the $i$-th atom, that is, 4 for Si atoms and 1 for $\mathrm{H}$ atoms.

The off-diagonal matrix elements (hopping parameters) of our Hamiltonian are calculated according to the Slater-Koster rules [23]. In this approach, the hopping parameters $t_{i \alpha, j \beta}$ are expressed as geometric functions of two-center integrals and depend only on the relative positions of the two centers $i$ and $j$. Contributions from three-center integrals are neglected. A detailed explanation of how to evaluate tunneling matrix elements was already published in Refs. [16,23]. Here the on-site energies $\varepsilon_{\alpha}$ and tunneling matrix elements $t_{i \alpha, j \beta}$ are not directly calculated, but obtained by fitting the TB band structure to the respective values 
measured experimentally or obtained by first-principles calculations. In this work, we use our own sets of TB parameters that fit the ab-initio DFT band structure of the passivated Si slab with reconstructed surface. More details about the optimized set of TB parameters are given in Sections IV and V.

\section{BAND STRUCTURE OF A Si BOX WITH HYDROGEN PASSIVATED AND RECONSTRUCTED SURFACE - A TIGHT BINDING MODEL}

In this section we report results and comparison of TB and DFT calculations of the band structure of the Si box (slab) shown in Fig. 1. In Fig. 2(a) we plot the energy bands of the model box calculated with ABINIT along the path defined by the symmetry points $\mathrm{G}=(0,0,0), \mathrm{Y}=\left(0, \pi / a_{2}, 0\right), \mathrm{S}=\left(\pi / a_{1}, \pi / a_{2}, 0\right)$ and $\mathrm{X}=\left(\pi / a_{1}, 0,0\right)$ of the surface Brilloin zone. The supercell shown in Fig. 1(a) has $16 \mathrm{Si}$ atoms and $6 \mathrm{H}$ atoms. With pseudopotentials accounting for core electrons, we have a total number of $N_{e}=70$ valence electrons that occupy the first 35 spin-degenerate lowest-energy bands. The Fermi level in Fig. 2 is indicated by the horizontal dashed red line. The energy gaps at the G, Y, S and X points obtained with DFT calculations are reported in Table【. We note that the DFT band structure in Fig. 2(a) reproduces very well the results reported recently by Bohloul et al. [24] using LDA and the projector augmented wave method implemented in VASP. [25] We

TABLE I. Energy gaps between the top of the valence band and the bottom of the conduction band $\left(E_{\text {gap }}\right)$ plotted in Fig. 2 calculated with DFT and TB methods at the points G, Y, S and X of the surface Brilloin zone. All values are given in $\mathrm{eV}$.

\begin{tabular}{lc|c}
\hline & DFT & TB \\
\hline$E_{\text {gap }}(\mathrm{G})$ & 1.144 & 1.656 \\
$E_{\text {gap }}(\mathrm{Y})$ & 2.262 & 3.078 \\
$E_{\text {gap }}(\mathrm{S})$ & 3.401 & 4.139 \\
$E_{\text {gap }}(\mathrm{X})$ & 1.592 & 2.496 \\
\hline
\end{tabular}



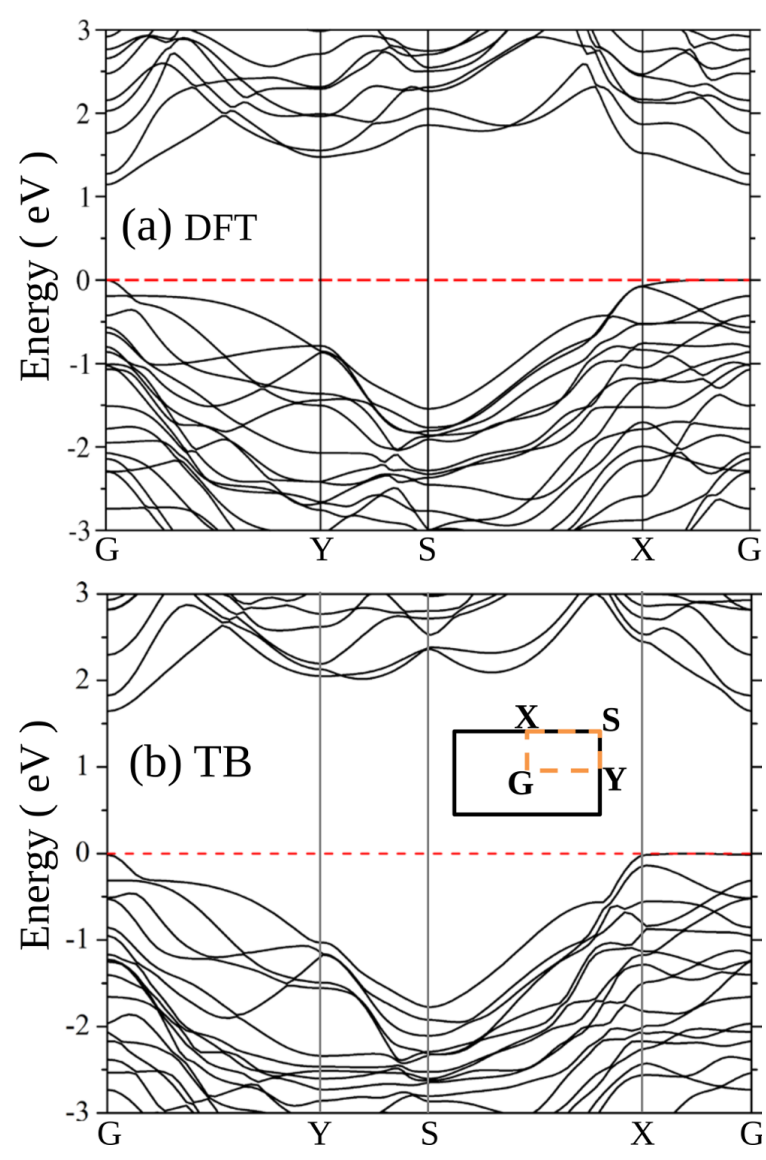

FIG. 2. (color online) Band structure of the reconstructed and H-passivated Si slab shown in Fig. 1 calculated with (a) DFT using GGA-PBE approximation and plane waves as implemented in the ABINIT program, and (b) the TB method with the set of parameters (see Table II) optimized to reproduce the ab-initio DFT band structure. The Fermi level is indicated with the dashed red line.

also note that DFT calculations performed within the LDA approximation by Wang et al. [26] predict a larger electronic gap of $2.0 \mathrm{eV}$ at the $\mathrm{G}$ point for a Si slab with a smaller lattice constant. The lower panel of Fig. 2 shows the energy bands for the same slab obtained by diagonalizing the TB Hamiltonian in Eq. (3) assuming periodic boundary conditions.

As we already mentioned, the surface reconstruction involves shifts of surface atoms from their bulk position and leads to the formation of a new Si-Si bond, absent in bulk. However, all Si atoms remain tetrahedrally coordinated and all bond lengths are remarkably close to the bulk nearest-neighbor separation. This is why for all Si atoms we utilize the TB parametrization of Klimeck et al. [27] obtained by fitting the TB model to reproduce 
TABLE II. Tight binding parameters for $\mathrm{Si}$ and $\mathrm{H}$ atoms on reconstructed surface used in the TB Hamiltonian to calculate the band structure shown in Fig. 2(b). All values are in eV.

\begin{tabular}{|c|c|c|c|c|c|}
\hline \multicolumn{3}{|c|}{ onsite energies } & \multicolumn{3}{|c|}{ hopping parameters } \\
\hline & $\mathrm{Si}$ & $\mathrm{H}$ & & $\mathrm{Si}-\mathrm{Si}$ & $\mathrm{Si}-\mathrm{H}$ \\
\hline$\varepsilon_{s}$ & -2.152 & -1.0 & $t_{s s}$ & -1.959 & -1.959 \\
\hline$\varepsilon_{p_{x, y}}$ & 4.229 & - & $t_{s p}$ & 3.026 & - \\
\hline$\varepsilon_{p_{z}}$ & 4.229 & - & $t_{p s}$ & 3.026 & 3.026 \\
\hline$\varepsilon_{d}$ & 13.789 & - & $t_{p p \sigma}$ & 4.104 & - \\
\hline \multirow[t]{11}{*}{$\varepsilon_{s^{*}}$} & 19.117 & - & $t_{p p \pi}$ & -1.518 & - \\
\hline & & & $t_{s d}$ & -2.285 & - \\
\hline & & & $t_{p d \sigma}$ & -1.355 & - \\
\hline & & & $t_{p d \pi}$ & 2.385 & - \\
\hline & & & $t_{d d \sigma}$ & -1.681 & - \\
\hline & & & $t_{d d \pi}$ & 2.588 & - \\
\hline & & & $t_{d d \delta}$ & -1.814 & - \\
\hline & & & $t_{s^{*} s}$ & -1.522 & - \\
\hline & & & $t_{s^{*} p}$ & 3.156 & - \\
\hline & & & $t_{s^{*} d}$ & -0.809 & - \\
\hline & & & $t_{s^{*} s^{*}}$ & -4.241 & - \\
\hline
\end{tabular}

the experimentally observed bulk Si band structure. The TB treatment of the $\mathrm{H}$ atoms passivating the top and the bottom Si surfaces is performed by choosing the Si-H hopping parameters identical to the Si-Si values, but adjusting the onsite energy of the $s$ orbitals on each $\mathrm{H}$ atom to obtain a good fit to the DFT data from Fig. 2(a). Values of all TB parameters are given in Table II. We note that we have repeated the calculation of the band structure accounting for the departures from bulk bond lengths and directions by scaling all TB matrix elements with strain corrections [27]. The resulting band structure was very similar to that shown in Fig. 2(b), and so we chose to utilize the bulk (unstrained) TB parametrization throughout this work. As can be seen from Fig. 2(b) this set of TB parameters captures all features of the band structure predicted by DFT. Our TB parametrization predicts larger 
electronic gaps for all symmetry points (see Table I). This is because the TB parameters were fitted to reproduce the experimental bulk Si bandstructure rather than that computed by DFT.

\section{DANGLING BOND WIRES ON A H:Si-(100)-(2×1) SURFACE}

If we remove a top $\mathrm{H}$ atom in the supercell shown in Fig. 3(a) and apply periodic boundary conditions, the resulting slab will have an array of DB wires (DBWs) along the row of Si dimers separated by a single wire of saturated bonds and $\mathrm{H}$ atoms as shown in Fig. 3(b). To validate our silicon computational box with reconstructed and passivated silicon surface, we compare here our DFT and TB results with DFT calculations reported in Ref. [24], where the same configuration was studied. Before this comparison, we note that DBWs suffer both Peierls distortion and dimerization, and/or antiferromagnetic ordering, as discussed by Lee et al. [28, 29]. These dimerization effects are specific to the one-dimensional nature of the nanowire and, for the time being, are beyond the scope of this work.

In Fig. 4(a) we show the results of DFT calculations of energy bands for the Si slab defined in Fig. 3. This supercell with a missing $\mathrm{H}$ atom has now an odd number of electrons $N_{e}=69$, hence we performed spin-polarized DFT calculations to obtain the band structure. We did not carry out the geometry optimization of the model slab. Such optimizations,

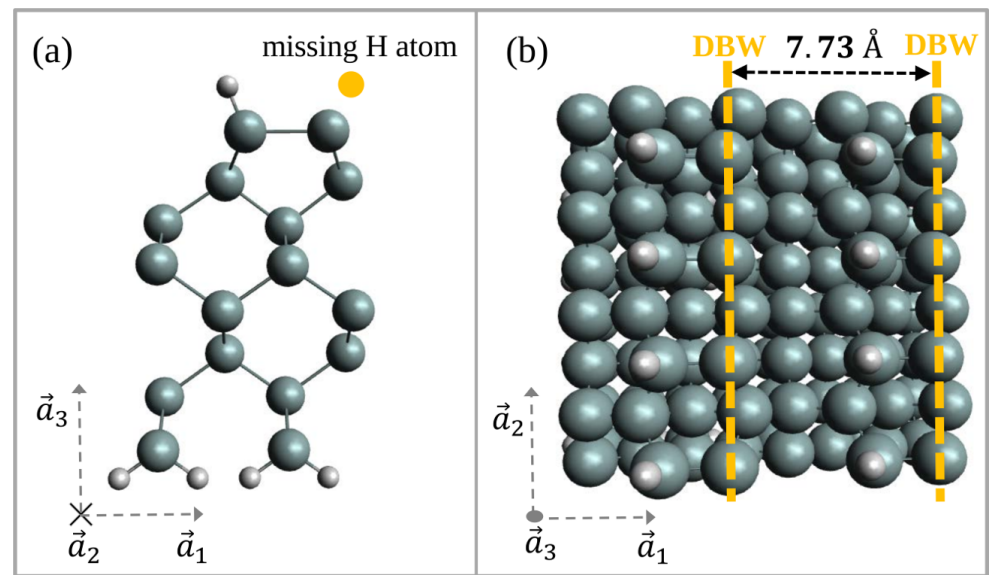

FIG. 3. (color online) (a) Side view of a supercell with a missing $\mathrm{H}$ atom generating an array of infinite dangling bond wires (DBWs). (b) Top view of a larger supercell illustrating the formation of DBWs on parallel dimer rows separated by the lattice constant $a_{1}=7.73 \AA$. 
performed by Watanabe et al. [30], revealed that the vertical position of the unsaturated $\mathrm{Si}$ atoms is lowered by only $0.1 \AA$. We used the Fermi-Dirac smearing to define the occupations of spin KS orbitals at each k-point during self-consistent calculations. Converged results show no spin polarization, with the highest occupied orbital equally populated by one half of the unpaired electron. This translates into identical band structures for the two spin components.

In Fig. 4(b) we plot the energy bands of the same Si slab obtained by solving the TB Hamiltonian, Eq. (3), with periodic boundary conditions. In our TB calculations, the removal of the Hydrogen atom is accounted for by i) setting the hopping parameters between the unsaturated $\mathrm{Si}$ atom and removed $\mathrm{H}$ atoms to zero, and ii) shifting the onsite energy of the $s$ orbital centered on this $\mathrm{H}$ atoms up in energy to avoid indirect coupling

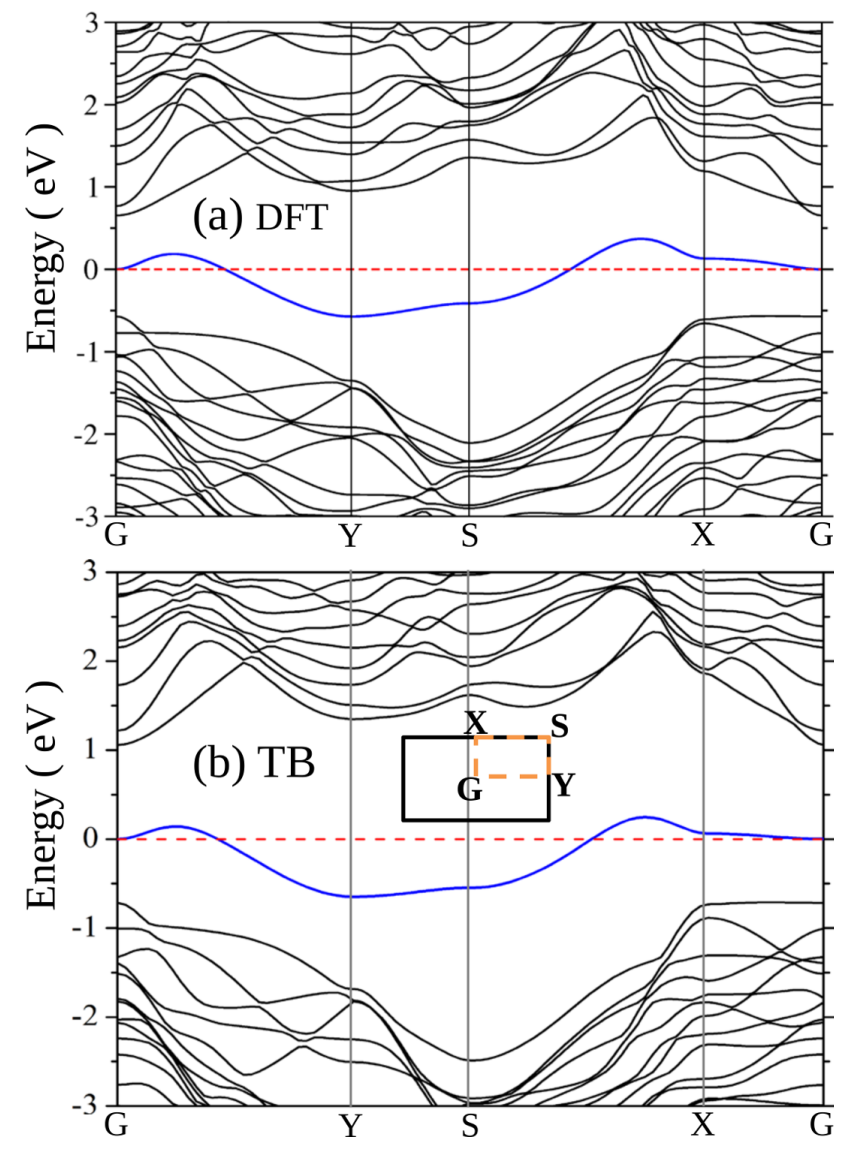

FIG. 4. (color online) (a) Energy band (in blue) in the Si bandgap associated with DBWs calculated with DFT (a) and with TB methods (b). The Fermi level is indicated with a dashed red line. 
TABLE III. Energy gaps between the top of the valence band and the midgap band of DB states $\left(E_{\text {gap }}^{\text {DB }}\right)$ at points G, Y, S and X as plotted in Fig. 4 calculated with DFT and TB. All values are given in $\mathrm{eV}$.

\begin{tabular}{lc|c}
\hline & DFT & TB \\
\hline$E_{\text {gap }}^{\mathrm{DB}}(\mathrm{G})$ & 0.570 & 0.716 \\
$E_{\text {gap }}^{\mathrm{DB}}(\mathrm{Y})$ & 0.778 & 1.036 \\
$E_{\text {gap }}^{\mathrm{DB}}(\mathrm{S})$ & 1.695 & 1.943 \\
$E_{\text {gap }}^{\mathrm{DB}}(\mathrm{X})$ & 0.738 & 0.795 \\
\hline
\end{tabular}

with other atoms. Furthermore, we adjusted the onsite energies of $s$ and $p$ atomic orbitals of depassivated Si atoms to the values $\varepsilon_{s}=-1.7 \mathrm{eV}$ and $\varepsilon_{p}=2.04 \mathrm{eV}$ to improve the TB description of the midgap band of DB states with respect to DFT results.

Both DFT and TB calculations show the emergence of a band of states in the Si bandgap, associated with the DBWs. This band, marked in blue, comprises electronic states localized on Si atoms with DBs on the surface. Note that this band shows a dispersion in all directions in reciprocal space due to coupling of DBs localized on different dimer rows. Furthermore, our DFT and TB calculations predict an energy-dispersion width for this band of $0.74 \mathrm{eV}$ which is in excellent agreement with previous calculations [10, 24, 30]. In Table III we report the energy difference between the top of the valence band and the DB states at the principal symmetry points. Note that TB calculations place these states at slightly higher energies as compared with analogous results calculated using DFT.

\section{DANGLING BOND QUANTUM DOT ON THE SILICON SURFACE}

We now turn to the description of a single DBQD on a passivated and reconstructed Si surface. With a single DBQD we abandon the periodic boundary conditions in all directions and develop a Si computational box ( $\mathrm{SiCB}$ ) properly passivated with $\mathrm{H}$ atoms on all sides, but with surface reconstruction only on the top side. Fig. 5 shows two computational 


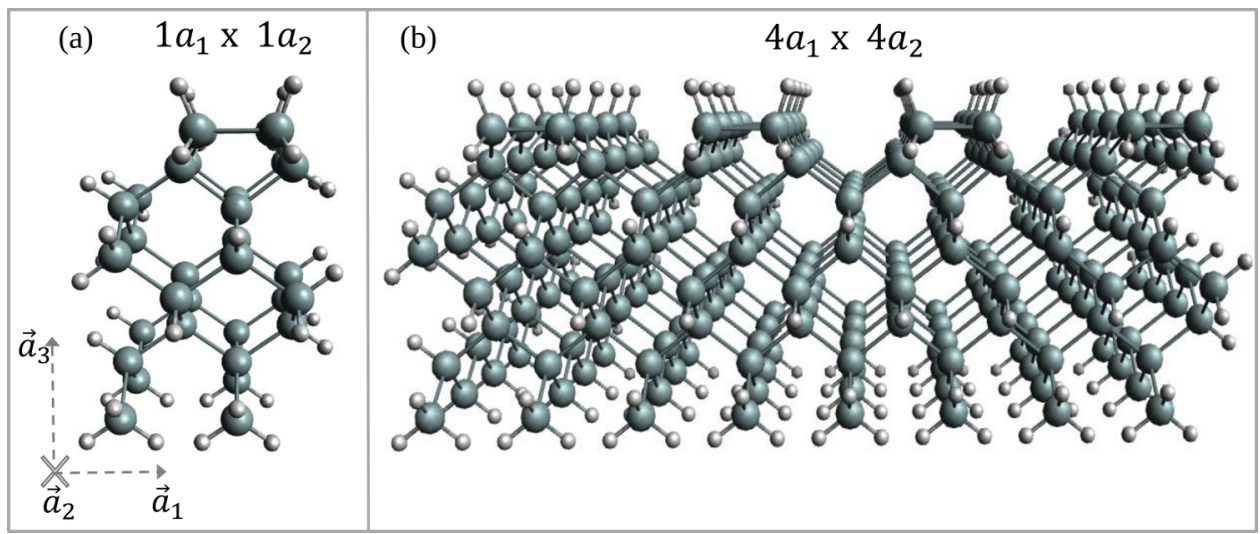

FIG. 5. (color online) Silicon computational box (SiCB) with top reconstructed surface consisting of (a) $N_{\mathrm{Si}}=32, N_{\mathrm{H}}=40, N_{e}=168$ and (b) $N_{\mathrm{Si}}=256, N_{\mathrm{H}}=184, N_{e}=1208$. The edge and bottom surfaces are passivated with $\mathrm{H}$ atoms without reconstruction. SiCBs are labeled based on the number of unit cells $(n)$ in the directions of $\vec{a}_{1}$ and $\vec{a}_{2}\left(n a_{1} \times n a_{2}\right)$.

boxes, labeled by the number of unit cells $(n)$ in the directions of $\vec{a}_{1}$ and $\vec{a}_{2}\left(n a_{1} \times n a_{2}\right)$. Fig. 5(a) shows the smallest possible slab of $N_{\mathrm{Si}}=32 \mathrm{Si}$ atoms passivated with $\mathrm{H}$. As already mentioned, the top layer is the reconstructed and passivated surface, while the unreconstructed bottom surface and edges are fully saturated with $\mathrm{H}$ atoms with the goal of simulating a seamless transition to the bulk Si. For $N_{\mathrm{Si}}=32 \mathrm{Si}$ atoms this requires $N_{\mathrm{H}}=40$ $\mathrm{H}$ atoms, with a total number of electrons $N_{e}=168$. In Fig. 5(b), a much larger SiCB, with $N_{\mathrm{Si}}=256, N_{\mathrm{H}}=184$, and $N_{e}=1208$, is shown.

Since these SiCBs are finite clusters, we carry out ab-initio DFT calculations using the OCTOPUs code [31] to solve the KS equations in a real-space representation. The real-space simulation domain was defined by using spheres centered at the atomic positions with radius $5 \AA$ and a uniform spacing of $0.19 \AA$ between each grid point of the generated mesh. We used atomic pseudopotentials to account for core electrons of Si atoms and the PBE-GGA approximation to the exchange-correlation potential. We followed these simulations by TB calculations with Si-QNANO with the parameters optimized to reproduce DFT energy bands of a Si slab as discussed in the previous sections.

Fig. 6 shows the results of DFT and TB calculations for the $N_{\mathrm{Si}}=32$ Si slab. Fig. 6(a) shows the energy levels of the slab fully passivated with H. Black bars show KS orbital energies calculated with OcTOPUs and red bars show the TB energy levels obtained with 


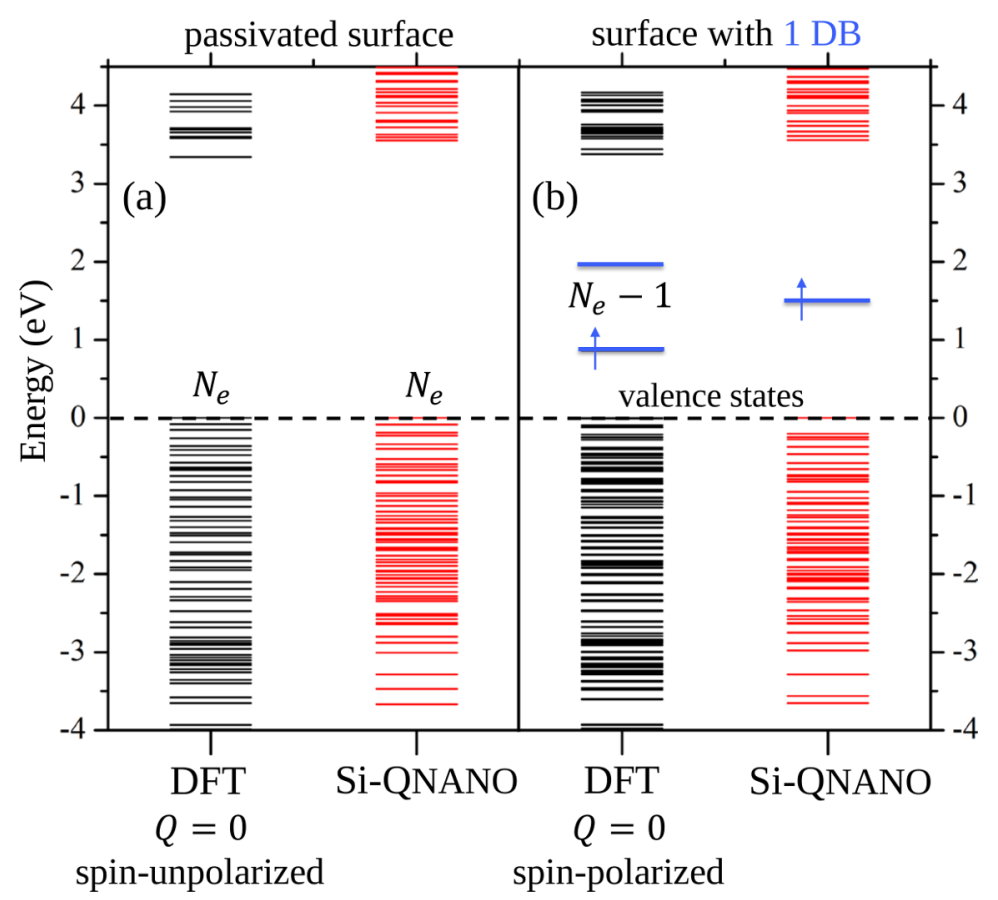

FIG. 6. (color online) (a) Energy levels of the Si computational box (SiCB) shown in Fig. 5(a) with the H-passivated top reconstructed surface. $Q$ denotes the net charge of the SiCB. (b) The effect of removal of a $\mathrm{H}$ atom from a top surface and a formation of a single dangling bond state with energy in the Si bandgap.

Si-Qnano. We fill the energy levels with spin up/down electrons up to the Fermi level which we take as reference energy level $E_{F}=0$. The structure is fully passivated, and we find the energy gap of $E_{\text {gap }} \approx 3 \mathrm{eV}$ opening in the energy spectrum in both DFT and TB calculations. Fig. 6(b) shows the effect of a removal of a $\mathrm{H}$ atom from the top surface, resulting in the formation of the DB. This implies a removal of one electron, and there are now $N_{e}=168-1$ electrons, with one electron on a Si DB. In addition, we characterize the cluster by the net charge $Q$. Because we removed both a proton and an electron, the net charge remains $Q=0$ even though there is a DB and an odd number of electrons.

The energy spectra for a surface with one DB show an emergence of an energy level, marked in blue, in the energy gap. As we have an odd number of electrons, in spin-polarized DFT the energy levels for spin up and spin down electrons are different. Thus, we have two localized levels that appear at $0.9 \mathrm{eV}$ and $1.9 \mathrm{eV}$ above the top valence state, respectively. In red we show the energy spectrum for the same Si cluster with a DB state obtained with 

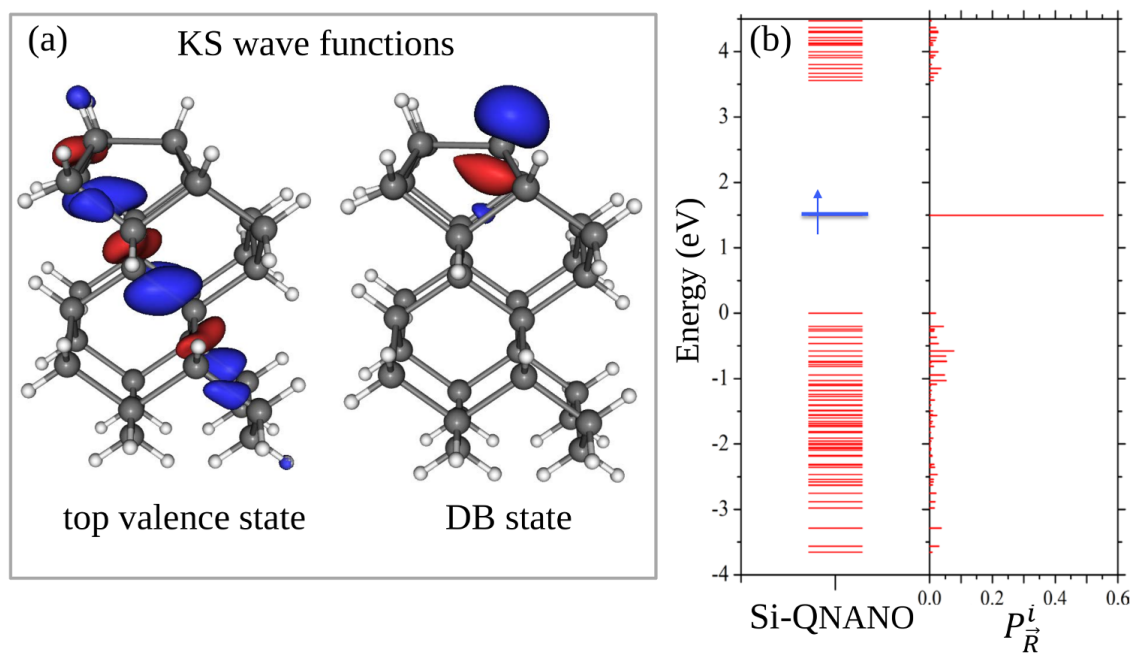

FIG. 7. (a) Isosurfaces of Kohn-Sham wave functions of the top valence state and the DB state with energy in the gap. (b) Energy levels of the SiCB $1 a_{1} \times 1 a_{2}$ shown in Fig. 5(a) and probability density $P_{\vec{R}}^{i}$ of finding the electron localized around the depassivated Si atom with position vector $\vec{R}$ calculated for each TB state $\left|\phi_{i}\right\rangle$.

Si-QNANO. We see that, just like in DFT calculations, there is a bound state, marked in blue, in the energy gap located at $1.5 \mathrm{eV}$ above the top valence state.

The localized nature of the DB state is shown in Fig. 7. In its left panel we visualize the KS wave functions of the top valence and DB states for the SiCB shown in Fig. 5(a). We find that while the valence state delocalizes over several Si atoms of the cluster, the wave function associated with the DB state is clearly localized around the Si atom with the DB. Furthermore, we also observe the $p_{z}$ atomic-like character of the DB KS orbital evident from the plotted isosurface. A similar scenario is confirmed by TB calculations. In Fig. 7(b) we plot the probability density $P_{\vec{R}}^{i}=\sum_{\alpha}\left|C_{\vec{R} \alpha}^{i}\right|^{2}$ of finding the electron localized around the Si atom with position vector $\vec{R}$ and with a $\mathrm{DB}$, calculated for each eigenstate $\left|\phi_{i}\right\rangle$. We see a distinct peak of $P_{\vec{R}}^{i}$ associated with the state in the energy gap indicating a strong electron localization on the Si atom with the DB.

In Fig. 8 we show the results obtained for a much larger computational box with $N_{\mathrm{Si}}=$ 256 and $N_{\mathrm{H}}=184$. We obtain qualitatively the same result. The energy gap in the spectrum appears, with the value $E_{\text {Gap }} \approx 2 \mathrm{eV}$, which is smaller than the energy gap for the smaller cluster as shown in Fig. 6. The removal of a $\mathrm{H}$ atom from the top surface results in the 

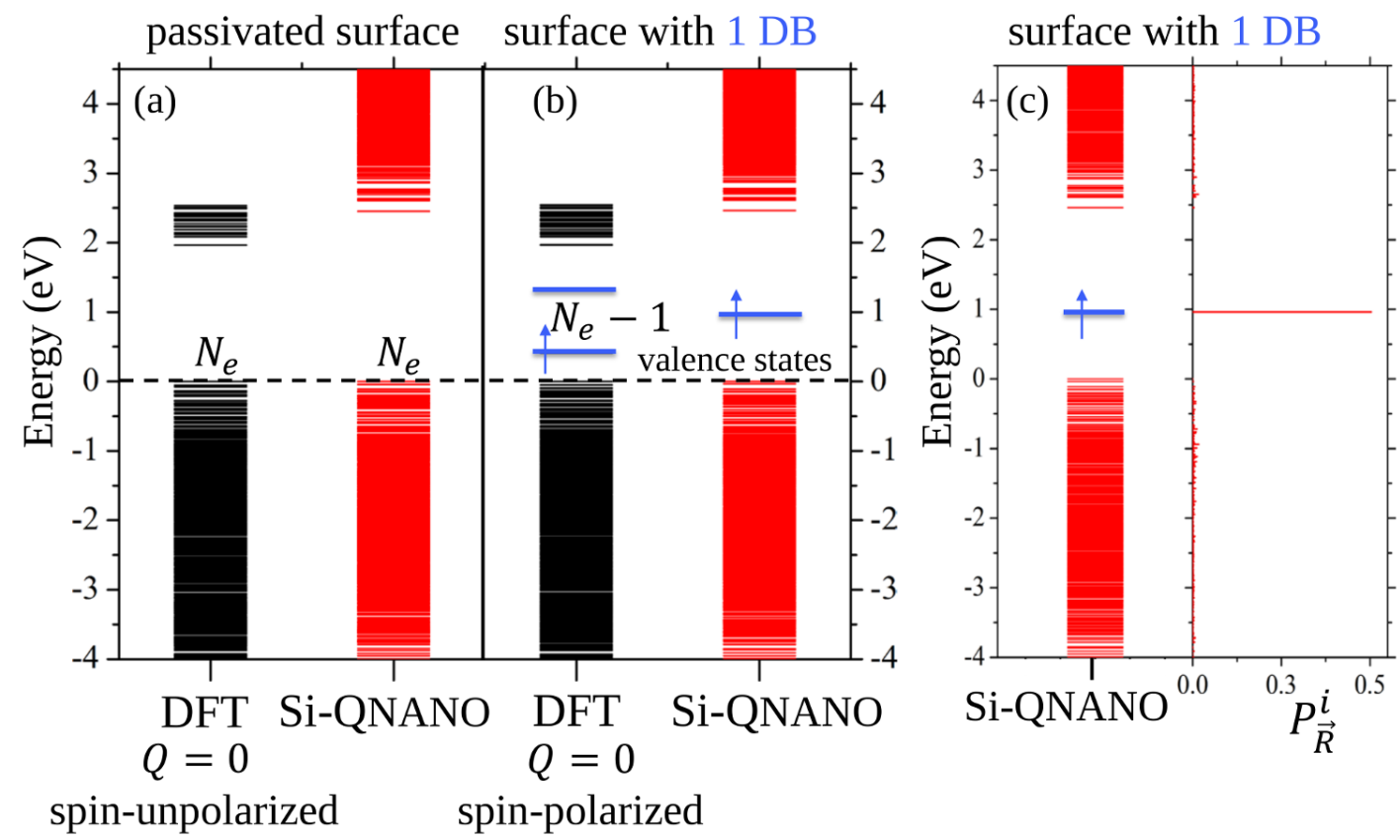

FIG. 8. (a) Energy levels of the $\operatorname{SiCB} 4 a_{1} \times 4 a_{2}$ shown in Fig. 5(b). $Q$ denotes the net charge of the SiCB. (b) The effect of removal of a $\mathrm{H}$ atom from the top surface and a formation of a single DB state with energy in the Si bandgap. (c) probability density $P_{\vec{R}}^{i}$ of finding the electron localized around the depassivated Si atom at position $\vec{R}$, calculated for each TB state $\left|\phi_{i}\right\rangle$.

appearance of a DB state in both DFT and TB spectra. Its energy is respectively $0.4 \mathrm{eV}$ and $0.9 \mathrm{eV}$ above the top valence state.

In Fig. 9 we plot the TB energy spectra calculated with Si-QNANO of Si computational boxes of increasing size with a single DB at the top surface. The simulated slabs consist of a total number of $\mathrm{Si}$ atoms $N_{\mathrm{Si}}$ ranging from 32 (the smallest possible cluster) to 14400 , a SiCB with dimension $30 a_{1} \times 30 a_{2}$. We used the Lanczos method, as implemented in SiQNANO, to calculate the energy levels of the finite slabs close to the top of the valence band and to the bottom of conduction band. In all cases a gap opens with the appearance of a DB state with the energy in the gap. Dashed red line in Fig. 9 shows the renormalization of the valence and conduction band edges as the size of the system increases. The energy gap decreases, as expected for a less confined system, and its value for the largest $\mathrm{SiCB}$, $E_{\text {Gap }}=1.6 \mathrm{eV}$, approaches the TB energy gap of an infinite slab as shown in Fig. 4. We also observe that the energy of the DB state converges very rapidly with the size of the Si slab due to the localized character of this state. For the largest $\mathrm{SiCB}$ with $N_{\mathrm{Si}}=14400$ atoms, 


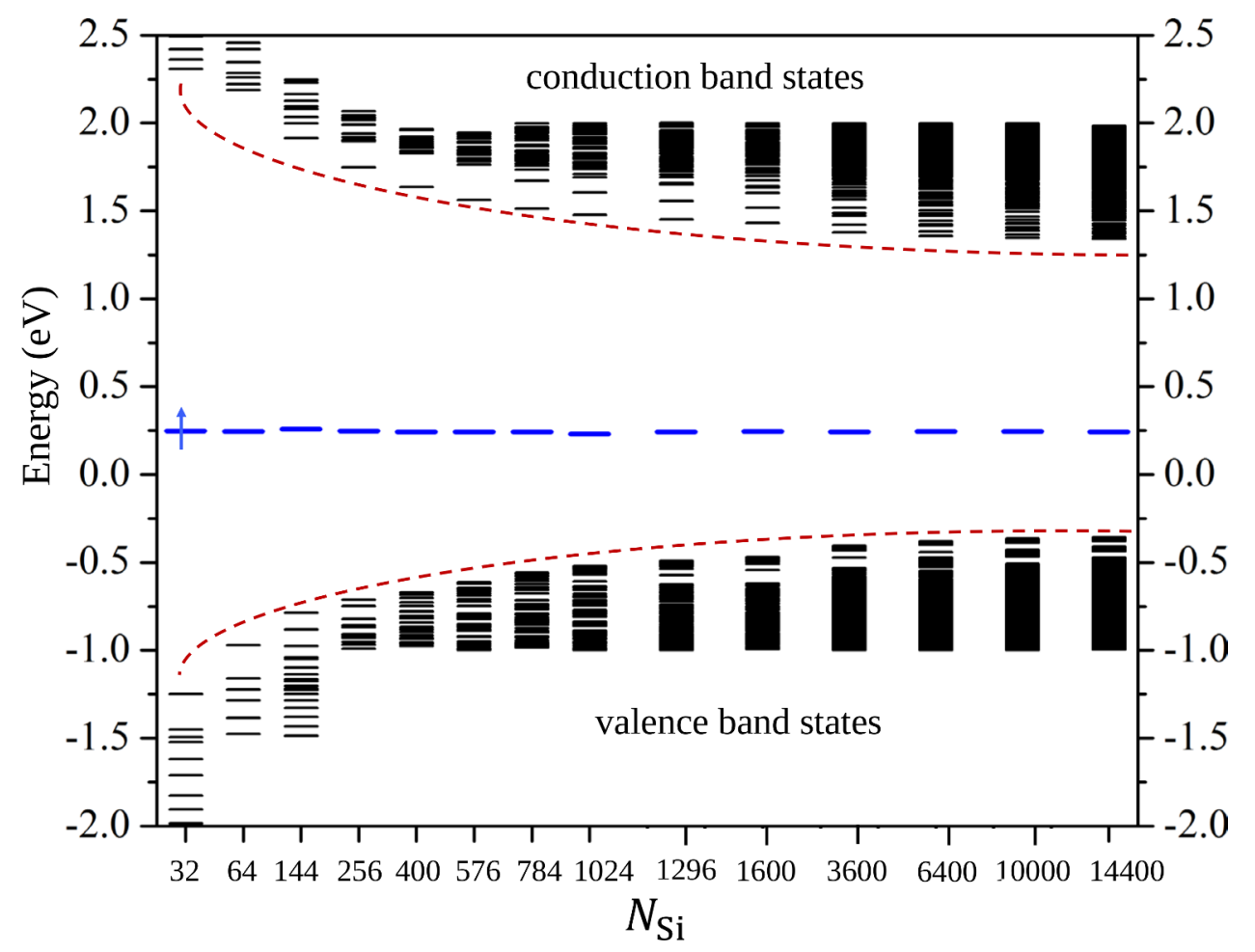

FIG. 9. (a) Energy levels of SiCBs of increasing size with a single DB in the top reconstructed surface calculated with Si-QNANO. The DB state is marked in blue, the arrow indicates singleelectron occupation of this state. Dashed red lines illustrate the evolution of the top of the valence band and the bottom of the conduction band with the size of the finite slab.

TB calculations with Si-QNANO place the DB state energy level $0.6 \mathrm{eV}$ above the top of the valence band.

\section{CHARGING ENERGY OF THE DANGLING BOND QUANTUM DOT}

STM experiments show that the dangling bonds simulated in the previous section are on average neutral, that is, the DB quantum dot is occupied by a single electron. However, for n-doped Si samples, additional electrons in the conduction band can be loaded into the DBQD in a controlled way using gates [6, 8]. We approximate the two-electron DBQD with a single configuration where two electrons with opposite spins are populating the DB orbital, with the net DBQD charge $Q=-1$. The DBQD charging energy $U_{\mathrm{DB}}$ is given by 
the two-body Coulomb matrix element,

$$
U_{\mathrm{DB}}=\int d \mathbf{x}_{1} d \mathbf{x}_{2} \phi_{u}^{*}\left(\mathbf{x}_{1}\right) \phi_{d}^{*}\left(\mathbf{x}_{2}\right) \frac{e^{2}}{\epsilon\left(\mathbf{x}_{1}, \mathbf{x}_{2}\right)\left|\mathbf{x}_{1}-\mathbf{x}_{2}\right|} \phi_{d}\left(\mathbf{x}_{2}\right) \phi_{u}\left(\mathbf{x}_{1}\right),
$$

where $\phi_{u}$ and $\phi_{d}$ are spin-up and spin-down DB spin-orbitals, each occupied by one electron at position $\mathbf{x} \equiv(\vec{r})$, and $\epsilon\left(\mathbf{x}_{1}, \mathbf{x}_{2}\right)$ is the position-dependent dielectric function. In order to evaluate this Coulomb matrix element we express the TB orbitals in the LCAO basis,

$$
\left|\phi_{i}\right\rangle=\sum_{\vec{R}, \alpha} C_{\vec{R} \alpha}^{i}|\vec{R} \alpha\rangle\left|\chi_{m_{\alpha}}\right\rangle
$$

with $\chi$ denoting the spin wave function. From Eq. (5) it follows that

$$
\begin{aligned}
U_{\mathrm{DB}}=\sum_{\vec{R}_{1}, \alpha_{1}} & \sum_{\vec{R}_{2}, \alpha_{2}} \sum_{\vec{R}_{3}, \alpha_{3}} \sum_{\vec{R}_{4}, \alpha_{4}} C_{\vec{R}_{1} \alpha_{1}}^{u^{*}} C_{\vec{R}_{2} \alpha_{2}}^{d^{*}} C_{\vec{R}_{3} \alpha_{3}}^{d} C_{\vec{R}_{4}, \alpha_{4}}^{u} \\
& \times \delta_{m_{\alpha_{1}}, m_{\alpha_{4}}} \delta_{m_{\alpha_{2}}, m_{\alpha_{3}}}\left\langle\vec{R}_{1} \alpha_{1}, \vec{R}_{2} \alpha_{2}\left|\frac{e^{2}}{\epsilon\left(\vec{r}_{1}, \vec{r}_{2}\right)\left|\vec{r}_{1}-\vec{r}_{2}\right|}\right| \vec{R}_{3} \alpha_{3}, \vec{R}_{4} \alpha_{4}\right\rangle .
\end{aligned}
$$

Equation (6) contains integrals up to four-center; in what follows We restrict the terms to one-center and two-center only. The integrals involving atomic orbitals centered on the same atom and on nearest-neighbor (NN) atoms are calculated numerically using Slater-type orbitals to approximate the radial part of the wave functions. The two-center integrals involving non-NN atoms are treated as long-range Coulomb interactions between distributions of two charges as explained in Ref. [16].

As we have shown in Figs. 7(b) and 8(c), for the DB state the probability density of finding the electron localized around the depassivated Si atom peaks at the large value of 0.6. This implies that the onsite term in Eq. (6) will give most of the contribution to the charging energy $U_{\mathrm{DB}}$ of the DB quantum dot. Indeed, we found that the DB charging energy shows practically no dependence on the size of the SiCB. On the other hand, the positiondependent dielectric function accounting for screening effects due to the valence electrons and the Si-vacuum interface has been taken in an approximate form [16]. More specifically, the on-site (dominant) contribution to the Coulomb interaction between the two electrons in the DB state is screened by the effective dielectric constant $\epsilon_{\mathrm{eff}}=\left(\epsilon_{\mathrm{Si}}+1\right) / 2$ where $\varepsilon_{\mathrm{Si}}=11.9$ is the Si static dielectric constant [22]. The smaller contributions involving atomic orbitals centered on neighboring atoms and non-NN atoms are screened by the dielectric constants $\epsilon_{\mathrm{Si}} / 2$ and $\epsilon_{\mathrm{Si}}$, respectively. 


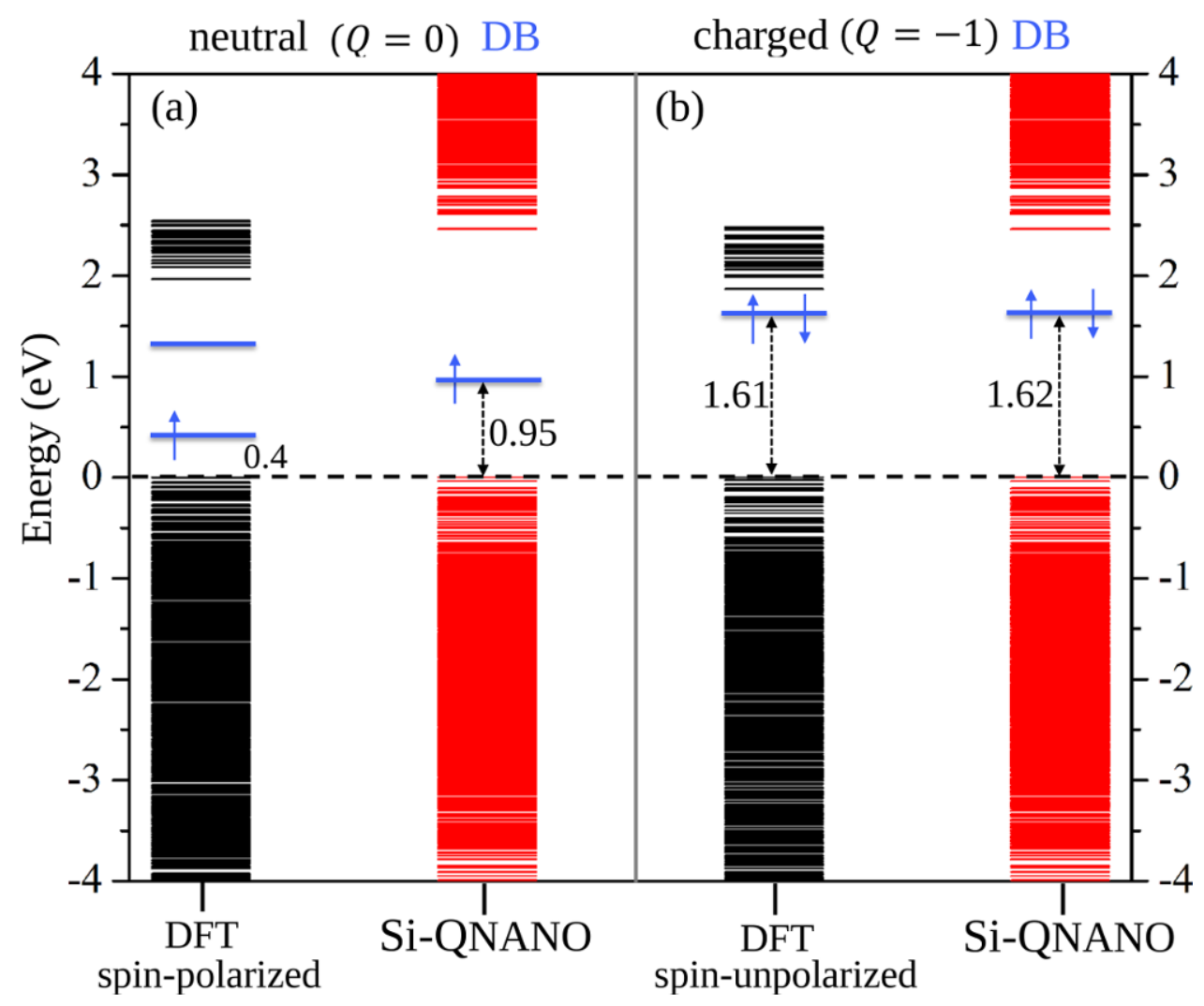

FIG. 10. Energy of the (a) neutral and (b) charged DB state for a SiCB $4 a_{1} \times 4 a_{2}$ shown in Fig. 5(b) with a depassivated Si atom at the top surface calculated with DFT and Si-QNANO.

Fig. 10 shows the energy spectra of the SiCB shown in Fig. 5(b) with a DB on the top surface calculated with DFT and the TB approach implemented by Si-QNANO. The DB states shown in Fig. 10(a) are occupied by one electron which corresponds to a neutral DBQD. On the other hand, Fig. 10(b) shows the energy spectra of SiCB with the net charge of $Q=-1$ where an extra electron with opposite spin populates the DB state. The DFT spectra are obtained by solving the spin-unpolarized KS equations for the negatively charged finite Si box. Note that in this case the KS energy of the DB state is shifted up by $1.2 \mathrm{eV}$ with respect to the energy of the neutral DB state. This means that each electron occupying the DB state for the system with $Q=-1$ experiences a $\mathrm{KS}$ effective potential due to the delocalized valence electrons plus a two-body Coulomb repulsion due to the presence of a second electron in the DBQD.

Similar conclusions hold for electrons occupying the TB states calculated with Si-QNANO. In this case, the energy of the DB state occupied by two non-interacting electrons will be 
shifted up in energy by the charging energy $U_{\mathrm{DB}}$ with respect to the singly-occupied $\mathrm{DB}$ state. In order to compare with DFT results, we used the TB wave function of the DB state to compute the charging energy using Eq. (6), for which we obtained the value of $0.67 \mathrm{eV}$. This places the energy of the negatively charged DB state at $1.62 \mathrm{eV}$ above the top of the valence band which is in excellent agreement with DFT results as shown in Fig. 10(b). In other words, our TB approach predicts a $0.67 \mathrm{eV}$ upward shift for the energy level of the charged DB relative to that of the neutral DB. We note that this number is in good agreement with the value of $0.5 \mathrm{eV}$ reported by Livadaru et al. 9]. Furthermore, the charged DB energy level predicted by Si-QNANO defines a bound state with energy -0.85 $\mathrm{eV}$ relative to the bottom of the conduction band which is similar to the value of $-0.95 \mathrm{eV}$ reported by Schofield et al. [11] to model STM experimental results on charged DB qdots. The small deviations of our results relative to the data reported by other authors may be related to the fact that we did not re-optimize the atomic position of the depassivated $\mathrm{Si}$ atom due to the excess charge localization.

\section{CONCLUSION}

We presented Si-QNANO, a new scalable computational platform to simulate atomic scale quantum devices in $\mathrm{Si}$. The central result is the construction of a $\mathrm{Si}$ computational box with a reconstructed surface opening the way toward realistic simulation of atomic scale devices on Si surface. We applied the Si-QNANO computational box to describe the dangling bond quantum dots on a hydrogen-passivated Si-(100)-(2x1) surface. The dangling bond due to the removal of a $\mathrm{H}$ atom was shown to result in an energy level localized in the Si bandgap, with wave function localized in the vicinity of the dangling bond silicon atom. The DBQD was shown to accommodate up to two electrons and the associated charging energy was predicted. For small number of $\mathrm{Si}$ atoms, the Si-QNANO results agreed very well with $a b$ initio calculations. However, Si-QNANO allowed us to compute the electronic properties for Di nanostructures involving tens of thousands of atoms. Future work will apply SiQNANO to dangling bond and dopant-based quantum circuits, gated quantum dots, and Si nanocrystals. 


\section{ACKNOWLEDGMENTS}

The authors thank R. Wolkow and K. Gordon of Quantum Silicon Inc., NSERC ENGAGE program and University of Ottawa Research Chair in Quantum Theory of Materials, Nanostructures and Devices for support.

[1] F. Priolo, T. Gregorkiewicz, M. Galli, and T. F. Krauss, Nature Nanotechnology 9, 19 (2014).

[2] T. F. Watson, S. G. J. Philips, E. Kawakami, D. R. Ward, P. Scarlino, M. Veldhorst, D. E. Savage, M. G. Lagally, M. Friesen, S. N. Coppersmith, M. A. Eriksson, and L. M. K. Vandersypen, Nature 555, 633 (2018).

[3] S. A. Studenikin, L. Gaudreau, K. Kataoka, D. G. Austing, and A. S. Sachrajda, Applied Physics Letters 112, 233101 (2018).

[4] S. Das Sarma, R. de Sousa, X. Hu, and B. Koiller, Solid State Communications 133, 737 (2005).

[5] J. Salfi, J. A. Mol, R. Rahman, G. Klimeck, M. Y. Simmons, L. C. L. Hollenberg, and S. Rogge, Nature Materials 13, 605 (2014).

[6] R. A. Wolkow, L. Livadaru, J. Pitters, M. Taucer, P. Piva, M. Salomons, M. Cloutier, and B. V. C. Martins, in Field-Coupled Nanocomputing (Springer, 2014) pp. 33-58.

[7] M. B. Haider, J. L. Pitters, G. A. DiLabio, L. Livadaru, J. Y. Mutus, and R. A. Wolkow, Physical Review Letters 102, 046805 (2009).

[8] M. Taucer, L. Livadaru, P. G. Piva, R. Achal, H. Labidi, J. L. Pitters, and R. A. Wolkow, Physical Review Letters 112, 256801 (2014).

[9] L. Livadaru, P. Xue, Z. Shaterzadeh-Yazdi, G. A. DiLabio, J. Mutus, J. L. Pitters, B. C. Sanders, and R. A. Wolkow, New Journal of Physics 12, 083018 (2010).

[10] P. Doumergue, L. Pizzagalli, C. Joachim, A. Altibelli, and A. Baratoff, Physical Review B 59, 15910 (1999).

[11] S. R. Schofield, P. Studer, C. F. Hirjibehedin, N. J. Curson, G. Aeppli, and D. R. Bowler, Nature Communications 4, 1649 (2013).

[12] M. Kepenekian, R. Robles, R. Rurali, and N. Lorente, Nanotechnology 25, 465703 (2014). 
[13] J. Wyrick, X. Wang, P. Namboodiri, S. W. Schmucker, R. V. Kashid, and R. M. Silver, Nano Lett. 18, 7502 (2018).

[14] H. Raza, Physical Review B 76, 045308 (2007).

[15] W. Sheng and P. Hawrylak, Physical Review B 72, 035326 (2005).

[16] M. Zielinski, M. Korkusinski, and P. Hawrylak, Physical Review B 81, 085301 (2010).

[17] M. Korkusinski, O. Voznyy, and P. Hawrylak, Physical Review B 84, 155327 (2011).

[18] J. P. Perdew, K. Burke, and M. Ernzerhof, Physical Review Letters 77, 3865 (1996).

[19] J. P. Perdew, K. Burke, and M. Ernzerhof, Physical Review Letters 78, 1396 (1997).

[20] X. Gonze, B. Amadon, P.-M. Anglade, J.-M. Beuken, F. Bottin, P. Boulanger, F. Bruneval, D. Caliste, R. Caracas, M. Cote, T. Deutsch, L. Genovese, P. Ghosez, M. Giantomassi, S. Goedecker, D. Hamann, P. Hermet, F. Jollet, G. Jomard, S. Leroux, M. Mancini, S. Mazevet, M. J. T. Oliveira, G. Onida, Y. Pouillon, T. Rangel, G.-M. Rignanese, D. Sangalli, R. Shaltaf, M. Torrent, M. J. Verstraete, G. Zerah, and J. Zwanziger, Computer Physics Communications 180, 2582 (2009).

[21] H. J. Monkhorst and J. D. Pack, Physical Review B 13, 5188 (1976).

[22] O. Madelung, Semiconductors: Data Handbook (Springer Science \& Business Media, 2012).

[23] G. F. Koster and J. C. Slater, Physical Review 95, 1167 (1954).

[24] S. Bohloul, Q. Shi, R. A. Wolkow, and H. Guo, Nano Letters 17, 322 (2017).

[25] G. Kresse and J. Furthmüller, Physical Review B 54, 11169 (1996).

[26] N.-P. Wang, M. Rohlfing, P. Krüger, and J. Pollmann, Physical Review B 74, 155405 (2006).

[27] T. B. Boykin, G. Klimeck, and F. Oyafuso, Physical Review B 69, 115201 (2004).

[28] J. Y. Lee, J.-H. Cho, and Z. Zhang, Physical Review B 80, 155329 (2009).

[29] J.-H. Cho and L. Kleinman, Physical Review B 66, 235405 (2002).

[30] S. Watanabe, Y. A. Ono, T. Hashizume, and Y. Wada, Physical Review B 54, R17308 (1996).

[31] X. Andrade, D. Strubbe, U. De Giovannini, A. H. Larsen, M. J. T. Oliveira, J. AlberdiRodriguez, A. Varas, I. Theophilou, N. Helbig, M. J. Verstraete, L. Stella, F. Nogueira, A. Aspuru-Guzik, A. Castro, M. A. L. Marques, and A. Rubio, Physical Chemistry Chemical Physics 17, 31371 (2015). 